\title{
Elaboración, caracterización y comparación de películas comestibles en base a aislado de proteínas de suero lácteo (WPI)
}

\author{
Escobar, D. (1), Márquez, R. (1), Repiso, L. (1), Sala, A. (2), Silvera, C. (2) \\ Contacto: rmarquez@latu.org.uy \\ (1) Gerencia de Proyectos Alimentarios. Investigación, Desarrollo e Innovación, Laboratorio Tecnológico del Uruguay \\ (LATU) - (2) Universidad Católica del Uruguay \\ Recibido: 26/02/2008 - Aprobado: 9/12/2008
}

\begin{abstract}
Resumen
El incremento del interés en alimentos de alta calidad con mayor vida útil y un menor impacto a nivel ambiental ha llevado al estudio y desarrollo de películas y coberturas comestibles y/o biodegradables. Éstas son una excelente alternativa de empaque de alimentos por su carácter biodegradable, flexible y su poder para actuar como envases activos. En base a esto y a la gran producción lechera en Uruguay, en el presente trabajo se elaboraron y estudiaron películas en base a WPI, como una alternativa de uso de los subproductos de la industria láctea de alto valor agregado.

Algunos plastificantes como glicerol y sorbitol modifican las propiedades de las proteínas insertándose dentro de su estructura tridimensional, y utilizados en pequeñas cantidades introducen cambios significativos.

El objetivo del presente trabajo fue la elaboración, caracterización y comparación de películas comestibles según sus propiedades mecánicas de tensión. Para ello se utilizaron dos concentraciones distintas de WPI (8\% y $10 \%)$ y tres proporciones de WPI/plastificante: $1,6 / 1 ; 2,0 / 1$ y $2,3 / 1$.

La disminución tanto de la cantidad de proteína como de plastificante en las películas produjo un aumento de las fuerzas y stress máximos y a la ruptura. Las películas más elásticas se obtienen con la mayor cantidad de plastificante estudiada (1,6/1), obteniéndose una elongación de hasta un $60 \%$ con un $8 \%$ de WPI en la película. Las películas realizadas con sorbitol presentan mayores fuerzas y tensiones que las de glicerol, manteniéndose en ambos casos la tendencia a la disminución de dichas propiedades con el aumento de plastificante. La elasticidad aumenta a mayor proporción de cualquiera de los plastificantes estudiados y las películas que contienen glicerol son más elásticas que las que contienen sorbitol.

Palabras clave: Película biodegradable, plastificante, fuerza, stress, elongación, módulo de Young.
\end{abstract}

\begin{abstract}
$\underline{\text { Abstract }}$
Increasing interest in high-quality food products with increased shelf life and reduced environmental impact has encouraged the study and development of edible and/or biodegradable films and coatings. These are an excellent alternative for food products packaging due to their biodegradable, flexible and active packaging characteristics. According to this and the large uruguayan milk production in this work WPI films were made and studied.

By other side, plasticizers as glycerol and sorbitol change the protein's properties because they easily insert within the threedimensional protein network, and used at low levels make significant changes.

The objective of the present work was to manufacture, characterize and compare edible films by their mechanical properties. For that two different WPI concentrations were used $(8 \%$ and $10 \%)$ and three different WPI/plasticizer ratios: 1,6/1; 2,0/1 and 2,3/1. The lower protein or plasticizer concentration in the films led an increase of maximum load, force at break and tensile strength at maximum and at break. The higher elongation of a $60 \%$ was obtained with the greater quantity of plasticizer (ratio 1,6/1) and with an $8 \%$ of WPI in the film. The mechanical properties of biofilms were compared using three ratios of WPI/plastizicer. The films made with sorbitol had bigger forces and tensile strength than those with glycerol, and with both plastizicers the higher quantity of them led a lower force and tensile strength. The elasticity reases to a greater proportion of any of the studied plastizicers. The films made with glycerol as plastizicer are more elastic than the sorbitol ones.

Key words: Edible film, plastizicer, force, stress, elongation, elastic modulus.
\end{abstract}

\section{Introducción}

Las películas comestibles están adquiriendo importancia como alternativa de empaque de alimentos por su carácter biodegradable y flexible, conjuntamente con la ventaja del agregado de aditivos que pueden mejorar la calidad y vida útil de los mismos. Los films producidos con aislado de proteínas lácteas (WPI) son excelentes candidatos por sus características funcionales y nutricionales, pues presentan buenas propiedades mecánicas y de barrera al oxígeno. Los plastificantes modifican las propiedades de las proteínas y en pequeñas cantidades introducen cambios significativos en las propiedades físicas y mecánicas de las matrices. Tanto el glicerol como el sorbitol reducen los enlaces internos de hidrógeno, aumentando el espacio intermolecular. Por este motivo se estudia y compara la influencia de ambos plastificantes en películas realizadas en base a aislado de proteínas de suero lácteo.

\section{Objetivos}

Elaboración, caracterización y comparación de films comestibles en base a dos concentraciones distintas de aislado de proteínas de suero lácteo (WPI), utilizando glicerol o sorbitol como plastificantes, en distintas relaciones WPI/plastificante. 
Objetivo específico 1: Elaboración, caracterización y comparación de films comestibles realizados a base de WPI y glicerol como plastificante, estudiando las propiedades mecánicas de tensión. Para estos estudios se tomaron como variables la concentración proteica del WPI (8 \% y $10 \%)$, las relaciones WPI/glicerol $(1.6 / 1 ; 2.0 / 1$ y $2.3 / 1)$ y dos rangos de espesores $(0.124 \pm 0.010 \mathrm{~mm}$ y $0.150 \pm 0.010 \mathrm{~mm})$.

Objetivo específico 2: Ídem objetivo específico 1, pero utilizando como plastificante sorbitol.

Objetivo específico 3: Comparación de las propiedades estudiadas en los films elaborados para el cumplimiento de los objetivos específicos 1 y 2 .

\section{Materiales y Métodos}

\section{Preparación de los films}

Los films se prepararon a partir de soluciones de WPI (proteína aislada de suero lácteo PROVON ${ }^{\circledR} 290,90 \%$ p/p de Glanbia Nutritionals, WI, USA) en agua destilada utilizando como plastificantes glicerol (J.T. Baker) y sorbitol (Roquette Vic S.A., Francia). Se prepararon soluciones al 8 $\%$ y $10 \%$ de WPI, y a cada una de ellas se le incorporó plastificante de manera tal de obtener las relaciones WPI/plastificante: 1,6/1; 2,0/1 y 2,3/1. Se agitaron una hora por agitación magnética, se ajustó el $\mathrm{pH}$ a 7,0 con solución de $\mathrm{NaOH} 2 \mathrm{~N}$ o HCl $2 \mathrm{~N}$, según el caso. Se colocaron en baño de agua a $83 \pm 1^{\circ} \mathrm{C}$, el tiempo necesario para lograr un buen entrecruzamiento de las proteínas sin llegar a su coagulación. Los tiempos empleados fueron 10 minutos para las soluciones al $8 \%$ de WPI y 7 minutos para las soluciones al $10 \%$ de WPI. Al final de dichos tiempos se ajustó el peso de manera de mantener las condiciones iniciales. Cada solución fue preparada por duplicado. Se colocaron las soluciones en placas de petri de poliestireno de $14 \mathrm{~cm}$ de diámetro interno (Greiner Bio-One), de forma de que no aparecieran burbujas y manteniendo constante la cantidad de sólidos. Se secaron los films durante 19 horas a $23 \pm 2{ }^{\circ} \mathrm{C}$ y $55 \pm 5 \% \mathrm{HR}$, se despegaron de las placas y se acondicionaron a temperatura y humedad de cuatro a siete días para la realización de todos los ensayos.

\section{Espesor de las películas}

Se midió con micrómetro digital Digi Thickness Tester, de Toyoseiki, Japón, con una resolución de $1 \mu \mathrm{m}$. Se expresó como el promedio de 10 medidas tomadas aleatoriamente en los films ya cortados y acondicionados para la realización del ensayo.

\section{Ensayos mecánicos}

Se realizaron de acuerdo a la norma ASTM D 882-02.

Se cortaron los films en forma de probetas de $10 \mathrm{~mm}$ de ancho con un cortador de muestras neumático (Toyoseiki de Japón). Se midieron los espesores según lo descrito y se separaron en dos grupos según su espesor $(0.124 \pm 0.010 \mathrm{~mm}$ y $0.150 \pm 0.010 \mathrm{~mm})$. Posteriormente se realizaron los ensayos de tracción en un equipo Shimadzu Autograph AG-2000A. Se utilizó una separación de mordazas de $50 \mathrm{~mm}$ y se aplicó una velocidad de $50 \mathrm{~mm} / \mathrm{min}$. Se realizaron de cinco a ocho ensayos para cada tipo de film preparado en cada uno de los rangos de espesores. De los ensayos de tracción realizados se determinaron los siguientes parámetros: fuerza máxima, fuerza a la ruptura, stress máximo, stress a la ruptura, porcentaje de elongación y módulo de Young.

\section{Resultados y Discusión}

\section{FILMS REALIZADOS CON GLICEROL COMO PLASTIFICANTE}

\section{Fuerza máxima y fuerza a la ruptura}

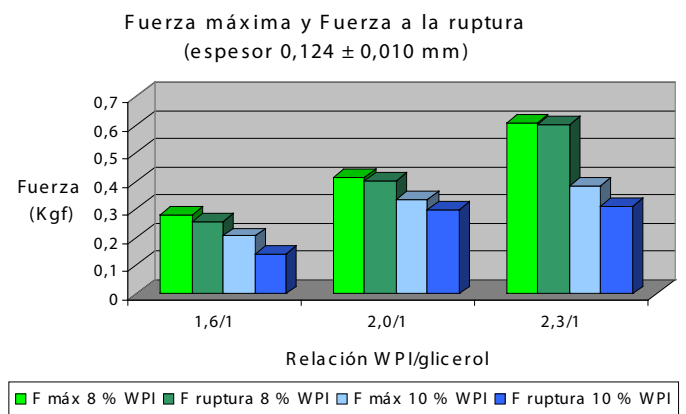

Gráfico 1. Comparación de fuerzas máximas y a la ruptura. Espesor $0.124 \pm 0.010 \mathrm{~mm}$.

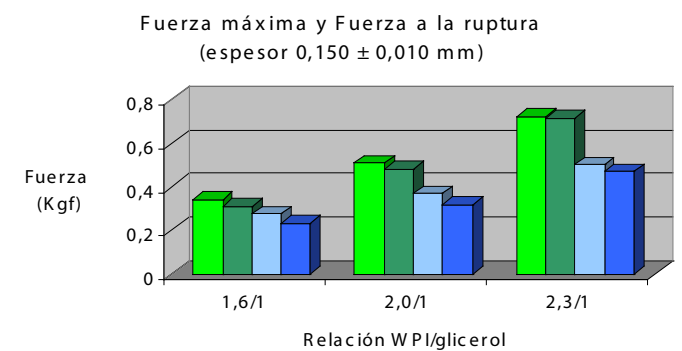

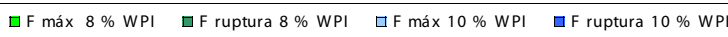

Gráfico 2. Comparación de fuerzas máximas y a la ruptura. Espesor $0.150 \pm 0.010 \mathrm{~mm}$.

- Tanto en el Gráfico 1 como en el Gráfico 2 se observa la tendencia a la disminución de las fuerzas máximas y de ruptura con el aumento del porcentaje de proteína en los films, manteniéndose en todas las relaciones WPI/plastificante.

Habiéndose realizado test de hipótesis al $95 \%$ de confianza ( $\mathrm{t}$ de student), se comprueba que son estadísticamente diferentes los valores en los casos estudiados con espesor $0.124 \pm 0.010 \mathrm{~mm}$ con relaciones de $1.6 / 1$ y $2.3 / 1$, y con los espesores $0.150 \pm 0.010$ en las relaciones 2.0/1 y 2.3/1. En los dos casos que no se pudieron comprobar estadísticamente los resultados (casos con espesor $0.124 \pm 0.010 \mathrm{~mm}$ de los films con $10 \%$ WPI y relación 2,3/1), puede haberse debido a que se trabajó con un número menor de muestras por rotura inadecuada de algunos films en los ensayos.

- En todos los casos se observa un aumento de las fuerzas máximas y de ruptura con el aumento de la relación WPI/glicerol, o sea con la disminución de plastificante.

Esto se comprueba estadísticamente al $95 \%$ de confianza en los casos estudiados, siendo la única excepción el de $10 \%$ WPI entre las relaciones $2.0 / 1$ y $2.3 / 1$ para el espesor de 0.124 $\pm 0.010 \mathrm{~mm}$, presumiéndose el mismo motivo mencionado anteriormente para este caso.

- La mayor fuerza soportada por los films estudiados fue de $0.72 \mathrm{Kgf}$ y se obtuvo con la relación $2.3 / 1 \mathrm{WPI} /$ glicerol y con un $8 \%$ de WPI, en el espesor de $0.150 \pm 0.010 \mathrm{~mm}$ [lo que concuerda con lo estudiado por Longares et al. (2004), Cuq et al. (1996) y Sobral (2000), quienes encontraron que la fuerza aumenta con el espesor del film]. 


\section{Stress máximo y stress a la ruptura}

Stress máximo y stress a la ruptura (espesor 0,124 $\pm 0,010 \mathrm{~mm}$ )

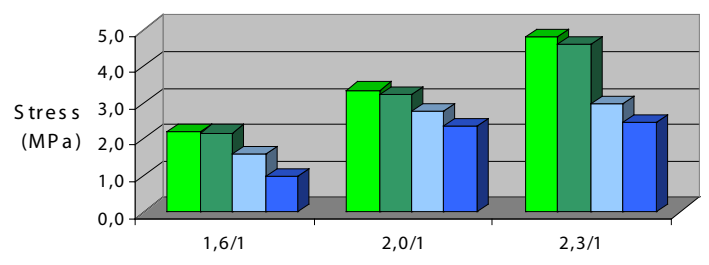

Relación W PI/glicerol stres s ruptura $8 \%$ WPI
stress ruptura $10 \%$ WPI

Gráfico 3. Comparación de stress máximo y a la ruptura. Espesor $0.124 \pm 0.010 \mathrm{~mm}$.

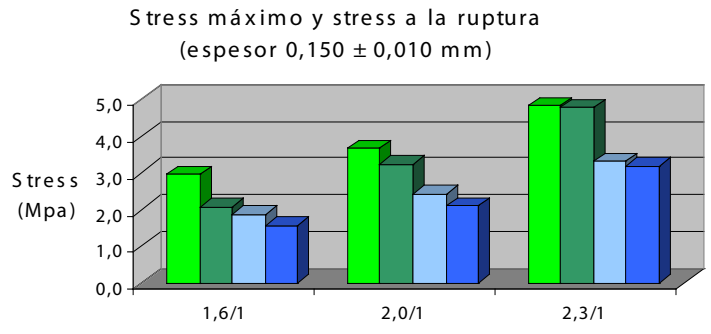

Relación W PI/glicerol

Gráfico 4. Comparación de stress máximo y a la ruptura. Espesor $0.150 \pm 0.010 \mathrm{~mm}$.

- En los Gráficos 3 y 4 se observa una tendencia a la disminución de los stress máximos y a la ruptura con el aumento del porcentaje de proteína en los films, manteniéndose en todas las relaciones WPI/ plastificante, al igual de lo que se vio para el estudio de las fuerzas máximas y de ruptura.

- En todos los casos se observa un aumento de los stress máximos y a la ruptura con el aumento de la relación WPI/glicerol, o sea con la disminución de plastificante.

Los estudios estadísticos para los stress desprenden los mismos resultados que para las fuerzas.

- El mayor stress soportado por los films estudiados fue de 4,8 MPa y se obtuvo con la relación 2.3/1 WPI/glicerol y con un $8 \%$ de proteína en ambos espesores analizados. Esto concuerda con lo hallado por Longares et al. (2004), donde se llega a la conclusión de que no hay diferencias significativas en los valores de stress con los distintos espesores. Esto puede ser atribuido a la inclusión del valor del espesor en los cálculos del stress, lo cual produce un efecto de normalización en los resultados con distintos espesores.

\section{Elongación}

Elongación

(espesor $0,124 \mathrm{~mm} \pm 0,010 \mathrm{~mm}$ )

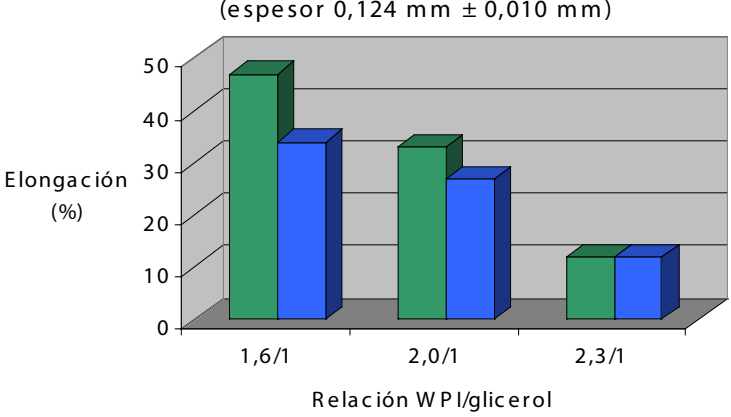

$\square 8 \% \mathrm{WPI}$ $\square 10 \%$ WPI
Elongación

(espesor $0,150 \mathrm{~mm} \pm 0,010 \mathrm{~mm}$ )

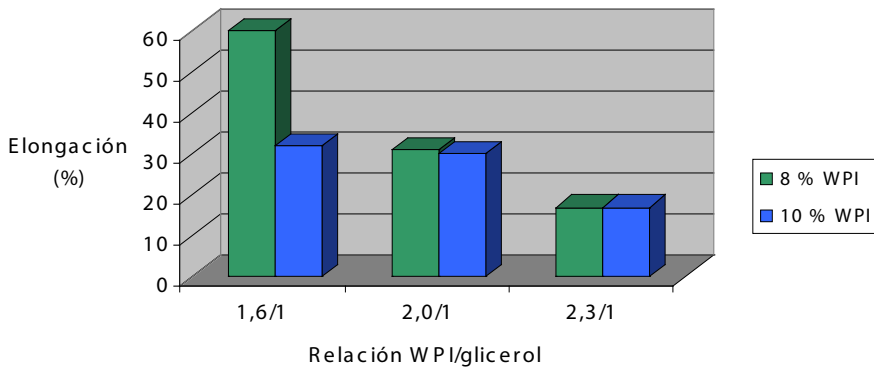

Gráfico 6. Comparación de elongaciones a la ruptura. Espesor $0.150 \pm 0.010 \mathrm{~mm}$.

- Entre los porcentajes de WPI de $8 \%$ y $10 \%$ no existen diferencias estadísticas al $95 \%$ de confianza en las elongaciones producidas, tal como lo muestran los Gráficos 5 y 6 . La única excepción se produce en los films con $8 \%$ WPI, relación $1.6 / 1$ y espesor 0.150 $+/-0.010 \mathrm{~mm}$

- Se presenta una tendencia a que los films con mayor cantidad de plastificante poseen mayor elongación. A esta misma conclusión arribó Sobral (2000). Esta tendencia se comprueba al $95 \%$ de confianza en todas las relaciones en los films con $8 \%$ WPI (espesor $0.124 \pm 0.010 \mathrm{~mm}$ ) y entre las relaciones $1.6 / 1$ y $2.0 / 1$ en los films con $10 \%$ WPI (espesor $0.150 \pm 0.010 \mathrm{~mm}$ )

- Los films más elásticos (con una elongación de un $60 \%$ ) fueron los films con $8 \%$ de WPI y con la mayor proporción de plastificante estudiados (1.6/1).

\section{Módulo de Young}

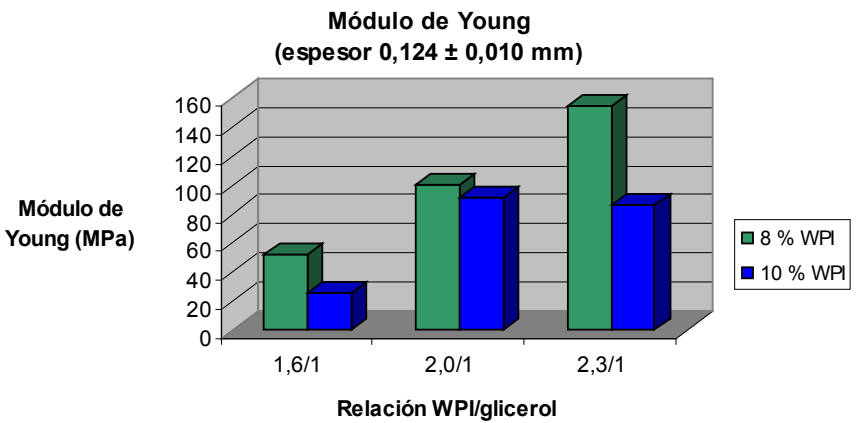

Gráfico 7. Comparación de módulos de Young. Espesor $0.124 \pm$ $0.010 \mathrm{~mm}$.

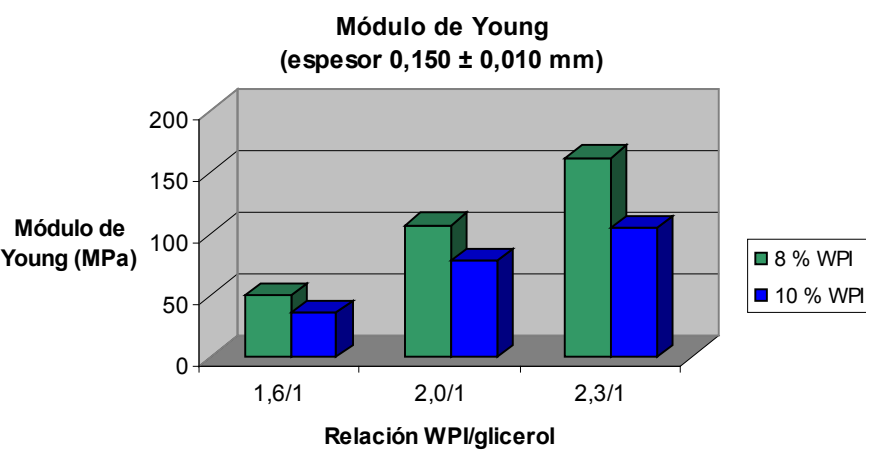

Gráfico 8. Comparación de módulos de Young. Espesor $0.150 \pm$ $0.010 \mathrm{~mm}$.

- En los Gráficos 7 y 8 se observa que a mayor proporción de plastificante (relación 1.6/1) el módulo de Young es menor, por lo que los films son más elásticos. Esta relación puede explicarse 
porque el glicerol reduce la interacción entre cadenas de proteínas e incrementa la flexibilidad, como lo deduce Sothornvit (2005).

- Dicha relación se comprueba en un $95 \%$ de confianza en los films analizados con espesor $0.150 \pm 0.010 \mathrm{~mm}$ y en los films al $8 \%$ WPI y todas sus relaciones con espesor $0.124 \pm 0.010 \mathrm{~mm}$.

- Se observan diferencias estadísticas entre los films con 8 y 10 $\%$ de WPI solo en las relaciones $1.6 / 1$ y $2.3 / 1$ de WPI/glicerol, siendo más rígidos los films con $8 \%$ de WPI (módulos de Young mayores).

\section{FILMS REALIZADOS CON SORBITOL COMO PLASTIFICANTE}

\section{Fuerza máxima y fuerza a la ruptura}

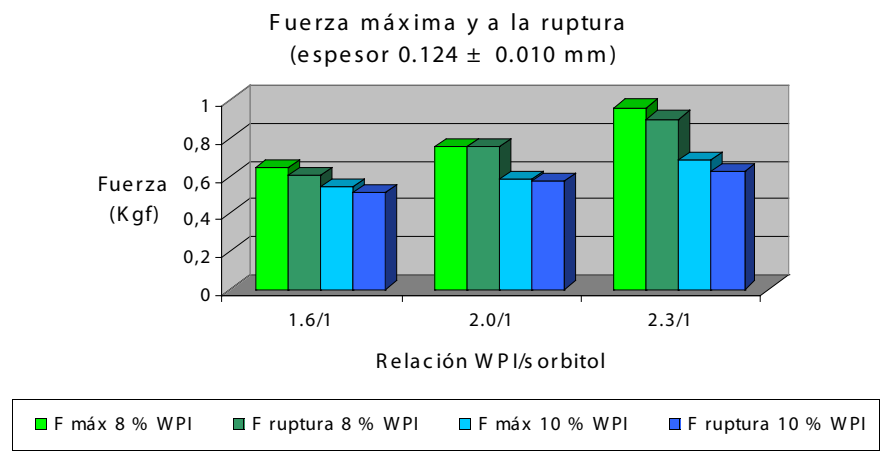

Gráfico 9. Comparación de fuerzas máxima y a la ruptura. Espesor $0.124 \pm 0.010 \mathrm{~mm}$

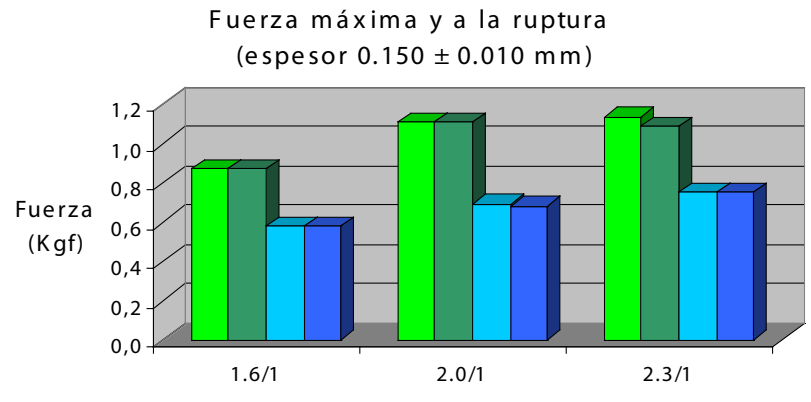

$\square$ F máx $8 \%$ WPI $\square$ F ruptura $8 \%$ WPI F máx $10 \%$ WPI q F ruptura $10 \%$ WPI

Gráfico 10. Comparación de fuerzas máxima y a la ruptura. Espesor $0.150 \pm 0.010 \mathrm{~mm}$.

- En los Gráficos 9 y 10 se puede observar una tendencia a la disminución de las fuerzas máximas y de ruptura con el aumento del porcentaje de WPI (del $8 \%$ al $10 \%$ ) en todos los films estudiados.

Solo se comprueba que son estadísticamente diferentes los valores al $95 \%$ de confianza en los casos estudiados con espesor 0.150 $\pm 0.010 \mathrm{~mm}$; y con espesor $0.124 \pm 0.010 \mathrm{~mm}$ únicamente en la relación 2.3/1. En los casos que no se pudieron comprobar estadísticamente los resultados, pudo haberse debido a altas desviaciones estándares en los resultados obtenidos.

Esta misma tendencia fue la que se obtuvo al utilizar glicerol como plastificante en las mismas relaciones y los mismos espesores.

- Al aumentar la proporción de plastificante (1.6/1), se observa una menor fuerza máxima y fuerza de ruptura.

Esto se comprueba estadísticamente con diferencias significativas a un $95 \%$ de confianza entre las relaciones $1.6 / 1$ y $2.3 / 1$ en ambos rangos de espesores cuando se utiliza un $8 \%$ de WPI, así como en el rango de espesores $0.150 \pm 0.010 \mathrm{~mm}$ se verifica diferencia estadística entre las relaciones $1.6 / 1$ y $2.0 / 1$ en el mismo porcentaje de proteína.

Esta misma tendencia se detectó al utilizar glicerol como plastificante utilizando las mismas relaciones y los mismos espesores. En el caso de glicerol, se obtuvo una comparación estadística más clara.

- La mayor fuerza máxima soportada por los films es de $1.14 \mathrm{Kgf}$ (espesor $0.150 \pm 0.010 \mathrm{~mm}$ ) para los films con $8 \%$ WPI y en la relación 2.3/1 WPI/sorbitol. Este resultado concuerda con lo concluido anteriormente, así como con la observación de la tendencia de mayores valores de fuerza a mayor espesor y con lo publicado por Longares (2004), Cuq et al. (1996) y Sobral (2000), quienes encontraron que la fuerza aumenta con el espesor (igual conclusión que para el caso estudiado con glicerol como plastificante).

\section{Stress máximo y stress a la ruptura}

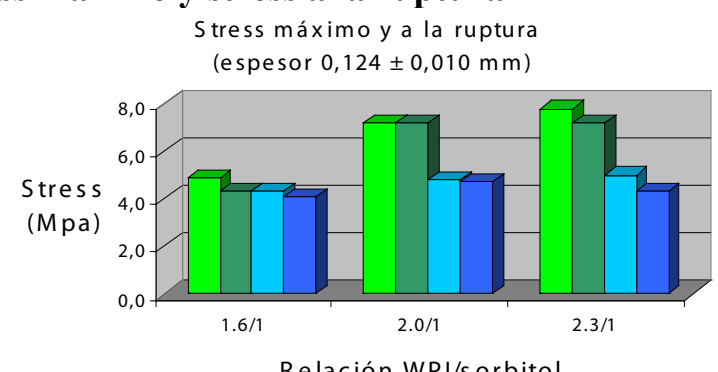

Relación WPI/sorbitol

$\begin{array}{ll}\text { 口 stress máx } 8 \% \mathrm{WPI} & \square \text { stress ruptura } 8 \% \mathrm{WPI} \\ \square \text { stress máx } 10 \% \mathrm{WPI} & \square \text { stress ruptura } 10 \% \mathrm{WPI}\end{array}$

Gráfico 11. Comparación de stress máximo y a la ruptura. Espesor $0.124 \pm 0.010 \mathrm{~mm}$

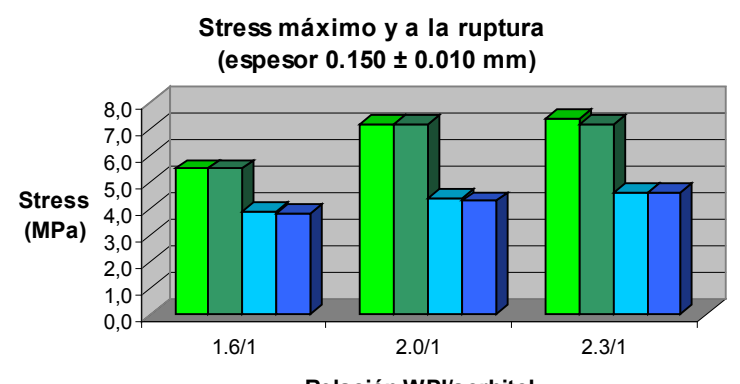

Relación WPI/sorbitol

$\begin{array}{ll}\text { 口 stress máx } 8 \% \mathrm{WPI} & \text { प stress ruptura } 8 \% \mathrm{WPI} \\ \text { प stress máx } 10 \% \mathrm{WPI} & \square \text { stress ruptura } 10 \% \mathrm{WPI}\end{array}$

Gráfico 12. Comparación de stress máximo y a la ruptura Espesor $0.150 \pm 0.010 \mathrm{~mm}$

- Los Gráficos 11 y 12 muestran que los resultados de stress mantienen la misma tendencia que los de las fuerzas, lo cual resulta lógico ya que el stress se define como la fuerza por unidad de área, manteniéndose estas últimas constantes.

Estadísticamente los comportamientos son similares a los obtenidos para las fuerzas.

- Esta misma tendencia del aumento de los stress con el aumento de la relación WPI/plastificante (o sea, con la disminución de la proporción de plastificante) la encuentran Shaw et al. (2002). Los autores atribuyen este hecho a que al incrementar la relación de plastificante (glicerol o sorbitol) disminuyen las interacciones intermoleculares en el entrecruzamiento de las proteínas. Doolittle (1965) sugiere que las moléculas de plastificante se enlazan con las moléculas de agua y con centros activos a lo largo de las cadenas de los polímeros, lo que hace decrecer las interacciones intermoleculares e incrementar las distancias intermoleculares. 
McHugh \& Krotcha (1994) reportan un decrecimiento de las tensiones con el aumento del plastificante con glicerol, pero no logran concluirlo para el sorbitol.

- El mayor stress soportado por los films estudiados fue de 7,8 MPa y se obtuvo con la relación 2.3/1 WPI/glicerol y con un $8 \%$ de WPI en los films con espesor $0.124 \pm 0.010 \mathrm{~mm}$. En el otro rango de espesores estudiado para esta misma relación se encontró un valor de stress máximo de 7,4 MPa, muy similar al mencionado anteriormente. Esta característica concuerda con lo encontrado por Longares (2004), donde se llega a la conclusión de que no hay diferencias significativas en los valores de stress entre los distintos espesores, lo cual puede ser atribuido a la inclusión del valor del espesor en los cálculos del stress, del mismo modo como se explicó para el caso de glicerol.

\section{Elongación}

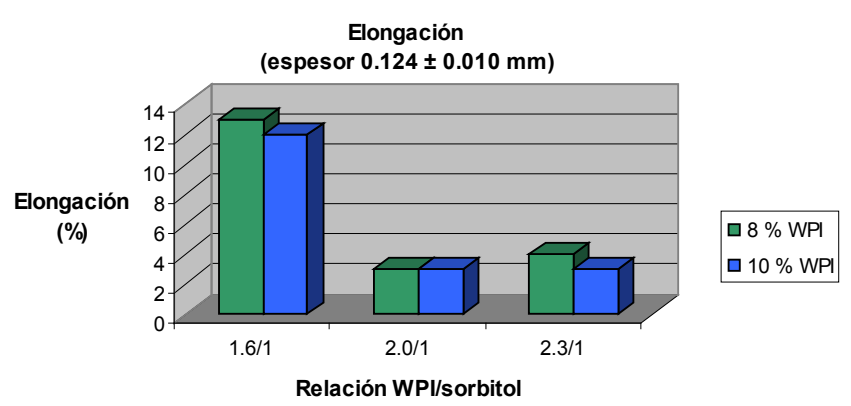

Gráfico 13. Comparación de elongaciones a la ruptura. Espesor $0.124 \pm 0.010 \mathrm{~mm}$.

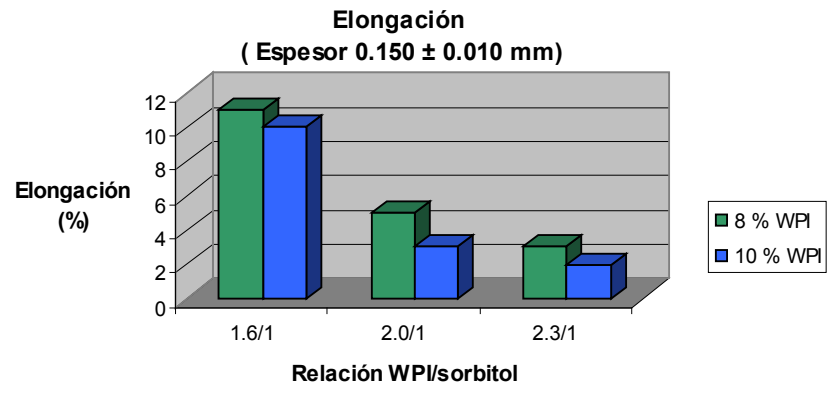

Gráfico 14. Comparación de elongaciones a la ruptura. Espesor $0.150 \pm 0.010 \mathrm{~mm}$.

- No se observan diferencias estadísticas en el porcentaje de elongación entre los films con $8 \%$ y $10 \%$ de WPI con un nivel de confianza de $95 \%$ en todas las relaciones WPI/sorbitol y espesores estudiados, como lo demuestran los Gráficos 13 y 14.

- Los films con mayor porcentaje de plastificante $(1,6 / 1)$, son los que poseen mayor elongación, comprobándose su diferencia estadística al $95 \%$ de confianza entre los de relación 2,0/1 y 2,3/1 de WPI/sorbitol, en los dos porcentajes de WPI estudiados. No se encontraron diferencias entre las elongaciones de los films con $2,0 / 1$ y $2,3 / 1$ estudiadas. Shaw, et al. (2002) y McHugh \& Krotcha (1994) encontraron que el porcentaje de elongación aumenta con el incremento de glicerol o sorbitol.

- El mayor porcentaje de elongación encontrado fue de $13 \%$ en los films con mayor proporción de plastificante, lo que indica un porcentaje relativamente bajo en relación a lo obtenido con los films con glicerol, donde utilizando las mismas condiciones se llegó a un 60 \% de elongación. Este resultado demuestra la poca elasticidad de los films realizados con sorbitol como plastificante.

\section{Módulo de Young}

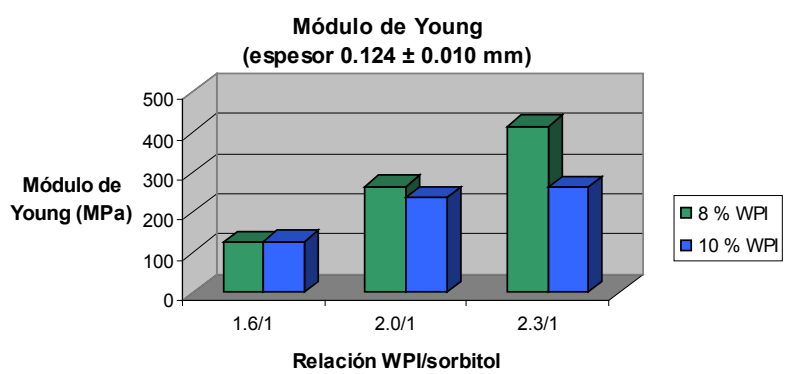

Gráfico 15. Comparación de módulos de Young. Espesor $0.124 \pm$ $0.010 \mathrm{~mm}$.

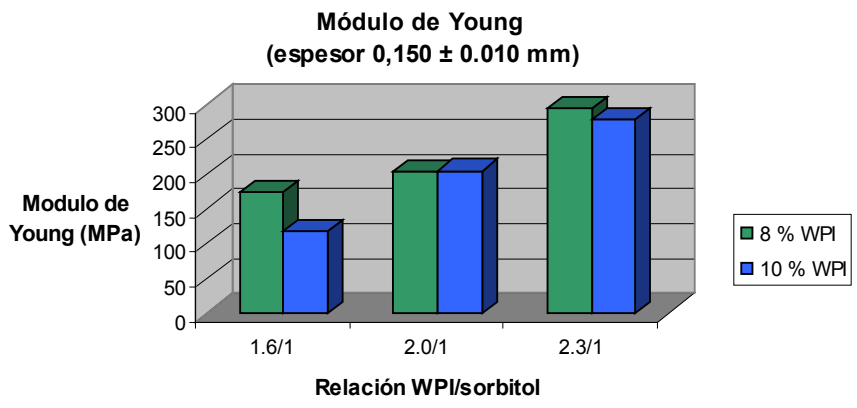

Gráfico 16. Comparación de módulos de Young. Espesor $0.150 \pm$ $0.010 \mathrm{~mm}$

- En los Gráficos 15 y 16 se puede observar una tendencia a que a menor relación WPI/sorbitol el módulo de Young es menor, por lo que los films son más elásticos. Del mismo modo Shaw et al. (2002) y McHugh \& Krotcha (1994) encontraron que el módulo de Young disminuye con el incremento de glicerol y sorbitol. Estos resultados se comprueban estadísticamente al $95 \%$ de confianza en casi todos los films de espesor $0.150 \pm 0.010 \mathrm{~mm}$, siendo la única excepción la diferencia entre las de relación 1,6/1 y 2,0/1 en los films que poseen $8 \%$ de WPI. En los films de espesor $0.124 \pm 0.010 \mathrm{~mm}$ la única excepción encontrada fue entre las relaciones $2,0 / 1$ y $2,3 / 1$ con un $10 \%$ de WPI.

- En la mayoría de los casos estudiados no se observan diferencias estadísticas entre los films realizados con $8 \%$ y $10 \%$ de WPI. Los casos que sí presentaron diferencias estadísticas al $95 \%$ de confianza fueron de relación 2,3/1 en los films de espesores 0.124 \pm 0.010 y los de relación 1,6/1 en el otro espesor estudiado.

- A menor módulo de Young, los films son más elásticos. Los menores valores encontrados fueron de $117 \mathrm{MPa}$ y $128 \mathrm{MPa}$ para los films realizados con una relación 1,6/1 WPI/sorbitol, lo que concuerda con que los mismos fueron los que presentaron mayor elongación (10 y $13 \%$, respectivamente).

\section{COMPARACIÓN DE GLICEROL Y SORBITOL COMO PLASTIFICANTES}

En este punto se comparan los resultados obtenidos anteriormente utilizando glicerol y sorbitol como plastificantes, en cada uno de los parámetros estudiados.

\section{Fuerzas máximas y a la ruptura}

- Los films realizados con sorbitol soportan mayor fuerza máxima y a la ruptura que los que tienen glicerol (del orden de 1.5 a 2.5 veces mayores).

Esto se comprueba estadísticamente al $95 \%$ de confianza en ambos porcentajes de WPI en casi todos los casos estudiados. Las 
excepciones fueron en los films con espesor $0.124 \pm 0.010 \mathrm{~mm}$ que se realizaron con la relación 2,0/1 de WPI/plastificante y con el espesor de $0.150 \pm 0.010 \mathrm{~mm}$ las $10 \%$ WPI y relación $2,3 / 1$. La no comprobación estadística en estos casos se debe a dos factores: el primero es que se rompieron algunos films en el ensayo, por lo cual el número de determinaciones realizadas fue menor, y el segundo fueron las altas desviaciones estándares que se obtuvieron.

- En ambos plastificantes se observa la tendencia de la disminución de las fuerzas máximas y de ruptura con el aumento del porcentaje de WPI (del $8 \%$ al $10 \%$ ) en todos los films estudiados.

- Asimismo, en ambos plastificantes se observa una tendencia al aumento de las fuerzas conforme disminuye la proporción de plastificante en los films.

\section{Stress máximo y a la ruptura}

- Los films con sorbitol soportan mayores stress máximos y a la ruptura que los de glicerol, tal como se concluye en el trabajo de Shaw et al. (2002).

Esto se comprueba estadísticamente al $95 \%$ de confianza en ambos porcentajes de WPI en casi todos los casos estudiados. Las excepciones fueron en los films con espesor $0.124 \pm 0.010 \mathrm{~mm}$ que se realizaron con $8 \%$ de WPI en la relación 2,0/1 de WPI/ plastificante; y los films con espesor $0.150 \pm 0.010 \mathrm{~mm}$ con $10 \%$ WPI y relación 2,3/1.

- En la comparación de stress se mantiene la misma tendencia que en las fuerzas, resultando la diferencia entre los stress de los distintos plastificantes mayores en los films de $8 \%$ que en los de $10 \%$ de WPI. También se observa un aumento de los stress al disminuir la proporción de plastificante, lo que resulta lógico ya que el stress se define como la fuerza por unidad de área, manteniéndose esta última constante.

\section{Elongación}

- Los films conteniendo glicerol poseen mayores elongaciones que los de sorbitol. Estas mismas conclusiones obtienen Shaw et al. (2002).

- En ambos plastificantes y espesores estudiados los films con mayor porcentaje de plastificante $(1,6 / 1)$, son los que poseen mayor elongación. Shaw et al (2002) y McHugh \& Krotcha (1994) encontraron que el porcentaje de elongación aumenta con el incremento de glicerol o sorbitol.

\section{Módulo de Young}

- Los films conteniendo sorbitol poseen mayores módulos de Young que los que tienen glicerol. Esto se comprueba en ambos porcentajes de proteínas y en todas las relaciones estudiadas. Estas mismas conclusiones obtienen Shaw et al. (2002).

Dado que este parámetro es una medida de la rigidez de los films, se puede decir que los films realizados con sorbitol son más rígidos, lo que concuerda con las bajas elongaciones obtenidas en relación al glicerol.

- En ambos plastificantes estudiados y en ambos espesores el módulo de Young aumenta con la disminución del porcentaje de plastificante.

Se observa que los films con menor proporción de plastificante (cualquiera de los dos usados) son los más rígidos. Esto concuerda con el hecho de que en ambos casos son los que presentan menor elongación.

\section{Conclusiones}

- Los films soportan mayor fuerza cuando: disminuye la proporción de plastificante, aumenta el espesor de los films y la concentración de proteína es del $8 \%$ para los dos tipos de plastificante estudiados.

- El stress tiene el mismo comportamiento que las fuerzas, en cuanto a que los mayores stress se obtienen con la disminución del porcentaje de plastificante y de la concentración de WPI. Sin embargo, esto no depende del espesor de los films, ya que existe un efecto de normalización por la inclusión del valor del espesor en los cálculos.

- Los films elaborados con sorbitol soportan mayores fuerzas y stress.

- Tanto los films elaborados con glicerol como con sorbitol presentan mayor elasticidad cuando hay una mayor proporción de plastificante, lo que se demuestra con las mayores elongaciones obtenidas y los valores más bajos de módulos de Young.

- Los films elaborados utilizando glicerol como plastificante son más elásticos que los de sorbitol. Esto lo demuestran tanto las elongaciones obtenidas como los módulos de Young que presentaron.

- Todas las tendencias observadas se mantienen en los dos espesores estudiados.

\section{Reconocimientos}

- LATU: Centro de Información, Departamento de Plásticos, Departamento de Metrología, Departamento de Microbiología.

- Glanbia Nutritionals Uruguay.

- Universidad de Sao Paulo: Facultad de Zootecnica e Engenharia de Alimentos, Pirassununga, SP, Brasil.

- PDT: Programa de Desarrollo Tecnológico.

- CNPq: Conselho Nacional de Desenvolvimento Científico e Tecnológico.

\section{Referencias}

- CUQ, B., et al. Functional properties of myofibrillar protein based biopackaging as affected by film thickness. En: Journal of Food Science. 1996, 61(3):580-584.

- DOOLITTLE, A. K. Mechanisms of plasticizatium. En: Bruins P. F. (ed.). Plasticizer technology. V. 1. New York: Reinhold Publishing, 1965. p. 1-20.

- KAYA, S.; KAYA, A. Microwave drying effects on properties of whey protein isolate edible films. En: Journal of Food Engineering. 2000, 43(2):91-96.

- LONGARES, A., et al. Physical properties and sensory evaluation of WPI films of varying thickness. En: Journal of Food Science and Technology. 2004, 37(5):545-550.

- MCHUGH, T.H.; KROTCHA, J.M. Milk-protein-based edible films and coatings. En: Food technology. 1994, 48(1):97-103.

- SHAW, N. B., et al. Physical properties of WPI films plasticized with glycerol, xylitol or sorbitol. En: Journal of Food Science. 2002, 67(1):164-167.

- SOBRAL, P.J. Thickness effects of myofibrillar protein based edible films on their functional properties. En: Pesquisa Agropecuaria Brasileira. 2000, 35(6):1251-1259.

- SOTHORNVIT, R., et al. Tensile properties of compressionmolded whey protein sheets: determination of molding condition and glycerol-content effects and comparison with solution-cast films. En: Journal of Food Engineering. 2006, 78(3):855-860 